\title{
Photo-graphies
}

\section{Les pratiques hybrides de Stéphane Couturier}

Entretien avec Monique Sicard

\section{Monique Sicard}

\section{CpenEdition}

Journals

\section{Édition électronique}

URL : http://journals.openedition.org/genesis/1503

DOI : 10.4000/genesis. 1503

ISSN : 2268-1590

Éditeur :

Presses universitaires de Paris Sorbonne (PUPS), Société internationale de génétique artistique littéraire et scientifique (SIGALES)

\section{Édition imprimée}

Date de publication : 15 avril 2015

Pagination : $99-115$

ISBN : 978-2-84050-992-9

ISSN : $1167-5101$

\section{Référence électronique}

Monique Sicard, «Les pratiques hybrides de Stéphane Couturier », Genesis [En ligne], 40 | 2015, mis en ligne le 13 avril 2017, consulté le 16 mai 2019. URL : http://journals.openedition.org/genesis/1503 ; DOI : 10.4000/genesis. 1503 


\title{
Les pratiques hybrides de Stéphane Couturier
}

\author{
Entretien avec Monique Sicard*
}

\begin{abstract}
Monique Sicard - J'ai connu vos travaux grâce aux photographies des usines Renault que vous aviez réalisées au début des années quatre-vingt-dix, alors qu' elles venaient de fermer. Avant leur démolition, nous ne connaissions d'elles, le plus souvent, que les façades grises de cette citadelle flottante de l'île Seguin. Vos photographies constituèrent un véritable événement en nous autorisant à visiter les lieux, désormais vides de toute présence humaine. À l'époque, ces images m'ont fascinée sans que je sache dire exactement pour quelles raisons. S'agissait-il de l'uniformité de la lumière, de la mise à plat d'espaces pourtant profonds ? La question restait posée : comment étiez-vous parvenu à produire de tels effets ? à l'aide de quels choix techniques, en obéissant à quelle intention, en suivant quelles étapes de réalisation? Les travaux de recherche entrepris désormais sur les processus de la création photographique permettent enfin d'interroger de vive voix l'auteur photographe.
\end{abstract}

\section{Billancourt}

Stéphane Couturier - De 1984 à 1992, j'ai effectué des reportages d'architecture destinés à des publications au sein de revues spécialisées. J'avais créé une agence de photo avec de nombreux photographes sensibles à l'architecture, comme Gabriele Basilico. Mais finalement, face à nous se trouvaient des architectes qui n'avaient pas envie d'une réflexion sur l'image. Nous avons fermé l'agence. Il ne s'agit pas d'un échec car les fonds existent dans une photothèque privée gérée par Françoise Morin, «Artedia ». Dans les années quatre-vingt-dix, j'avais commencé à travailler pour diverses commandes du CAUE921. Répondre à celle qui concernait Billancourt m'a permis finalement de me délivrer de ces contraintes et de reprendre ma liberté. J'ai travaillé en 1992-1993 sur l'extraordinaire enchevêtrement de l'architecture industrielle de cette ville dans la ville qu'étaient les usines Renault avant leur démolition.

J'ai beaucoup travaillé sur les paysages de la boucle de la Seine entre Sèvres et Issy-les-Moulineaux, notamment par la confrontation d'une vue depuis une rive du fleuve avec le contrechamp réalisé depuis l'autre rive. Mais je souhaitais accéder à l'intérieur de l'Île Seguin. Cela fut compliqué et m'a demandé beaucoup de temps ; les responsables de Renault n'étaient pas prêts à laisser entrer un photographe dans des bâtiments marqués par leur côté vieillot, déglingué. Ils ne souhaitaient pas communiquer sur une usine devenue obsolète. Il a fallu batailler et surtout, faire des photos qui n'étaient pas voulues. J'ai pris à bras-le-corps la commande du CAUE. Je suis même allé au-delà, retournant sur les lieux par moi-même, plus tard, un certain nombre de fois car le sujet me fascinait. En 2003, notamment, j'ai pu réaliser des images juste avant la démolition des lieux.

* Cet entretien a été produit dans le cadre du programme de recherche Photopaysage (ANR-13-BSH3-0008-01).

1. Conseil architecture et urbanisme des Hauts-de-Seine. 
$\begin{array}{llllllll}\text { G } & \text { E } & \text { N } & \text { E } & \text { S } & \text { I } & \text { S }\end{array}$

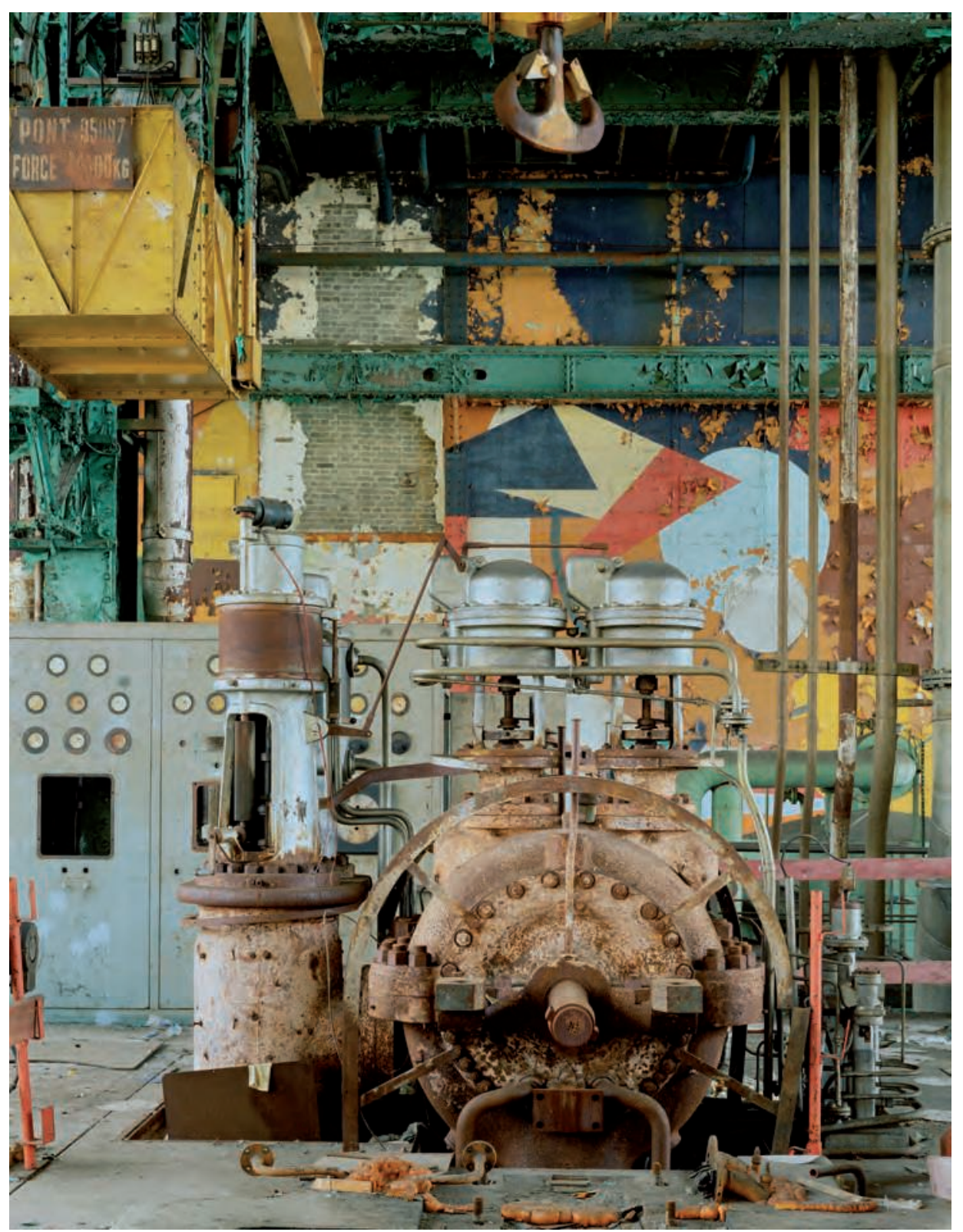

Fig. 1 : Renault, Île Seguin, 2003

(Ilfoflex, $160 \times 125 \mathrm{~cm}, 5$ exemplaires)

(c) Stéphane Couturier

J'aurais aimé savoir qui est l'auteur de la fresque sur le mur, derrière (fig. 1), mais personne ne le savait chez Renault. Ici, j'ai certainement été influencé par des peintres, tel Fernand Léger. L'industrie est génératrice de choses superbes.

\section{Villa Noailles I}

Vous avez réalisé des photographies de la villa Noailles, à Hyères, construite par Robert Mallet-Stevens. Elles jouent plus encore peut-être que les images de Billancourt sur la confusion qui s'opère entre les différents plans. Il est difficile de dire ce qui se trouve au premier plan, ce qui se trouve à l'arrière-plan (fig. 2). Il nous semble avoir affaire à un collage.

Je travaille avec une chambre $4 \times 5$ inches. $\mathrm{Ce}$ n'est pas une chambre de très grand format, mais un appareil de qualité. J'ai fait, depuis longtemps, le choix de la frontalité de la prise de vue, éliminant ainsi les obliques de perspective, redressant les verticales, calant 


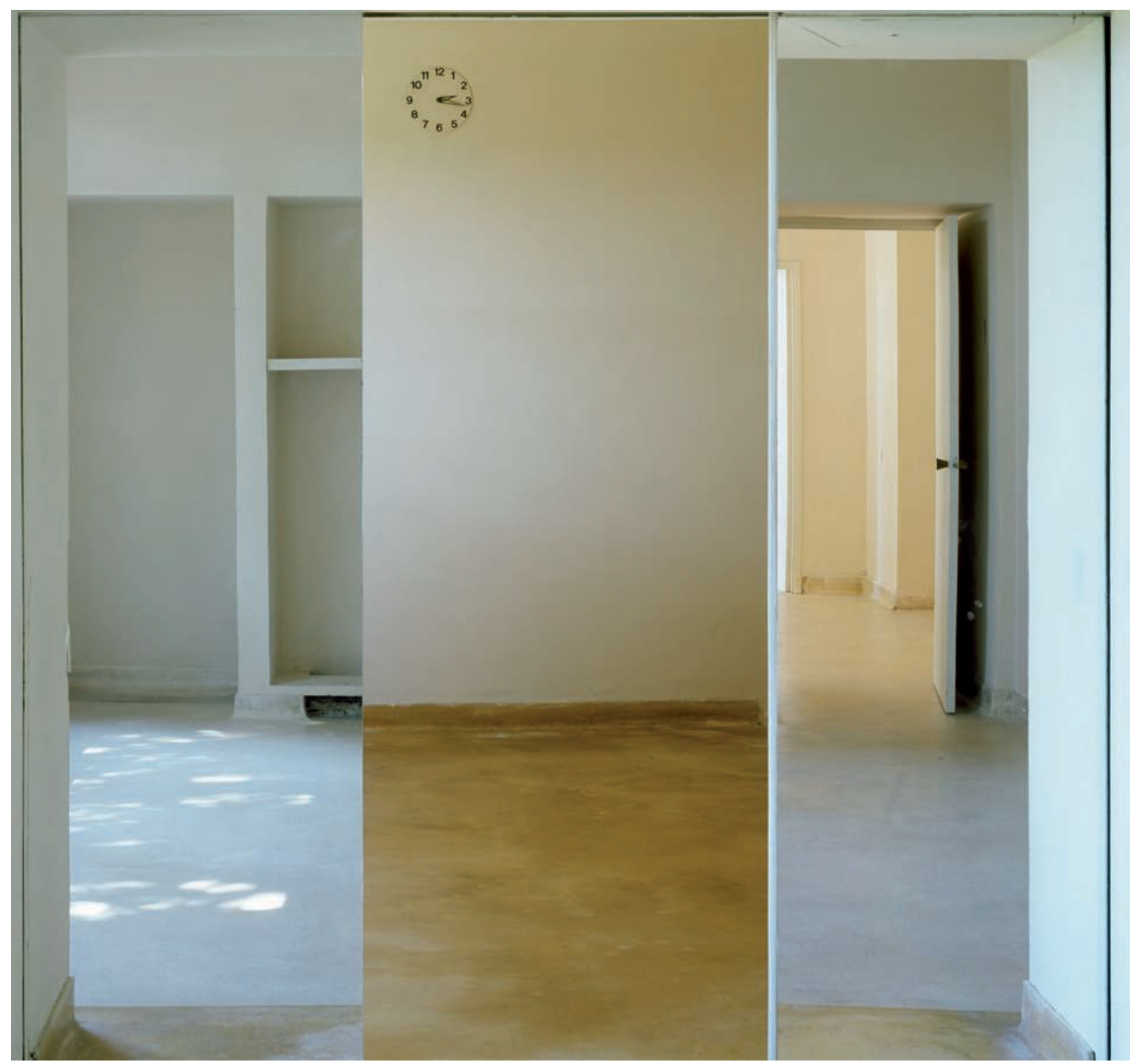

Fig. 2 : Villa Noailles, Hyères, 1996

(photo ${ }^{\circ} 6$, Ilfochrome, $112 \times 118 \mathrm{~cm}, 3$ exemplaires)

(c) Stéphane Couturier

les horizontales. La chambre permet cela en facilitant les repérages sur le verre dépoli. Au départ, ce choix de la frontalité n'était pas intentionnel. Je ne me suis aperçu que par la suite qu'il irriguait l'ensemble de mes photographies.

La qualité des tirages joue certainement un rôle très important dans la réception que nous avons de ces images. Dans quelle mesure leur matière, leur velouté, le jaune profond de ces photographies de la villa Noailles tiennent-ils aux choix des procédés de tirage?
Toutes les photos, celles de la villa Noailles comme les autres, ont été prises à la chambre. Il s'agit toujours d'un travail en positif couleur. Cela permet une visualisation et une réponse plus rapide qu'avec des films négatifs. Ces films positifs sont ensuite tirés sur Cibachrome, papier que l'on ne peut utiliser que si l'on travaille avec des diapositives couleur. Le tireur, Roland Dufau, a réalisé un certain nombre de mes tirages Cibachrome, mais ses machines ne sont pas utilisables au-dessus du format $60 \times 80 \mathrm{~cm}$. Comme je me situais la plupart du temps dans de 
plus grands formats, j'étais obligé de me tourner vers l'atelier Publimod et son tireur, Choi. Roland Dufau, comme Choi, a heureusement anticipé la fermeture de l'usine suisse qui fabriquait le Cibachrome en faisant des stocks de papier. À l'heure numérique, je trouve intéressant de travailler avec ces papiers argentiques qui possèdent une certaine noblesse, une pérennité et des qualités que l'on ne trouve pas dans les nouveaux papiers.

Certaines de mes photographies sont présentées sous Plexiglas transparent, du type Diasec (résine acrylique). La qualité de la pénétration et la réfraction de la lumière dans ce verre acrylique rendent les couleurs plus brillantes et l'image plus nette que sous un verre standard. Habituellement, le tirage est collé bien à plat sur ce verre acrylique. Mais quand je présente ces photographies non contrecollées, à l'intérieur d'un cadre, cela leur permet de flotter un peu, de conserver visiblement une certaine matérialité. Je suis passé au tirage numérique, mais je songe à retravailler avec du papier Cibachrome : on obtient un beau velouté et des couleurs intéressantes. Voyez ce vert ! Je me rends compte également qu'un certain nombre de collectionneurs veulent avoir du papier Cibachrome. Il est possible en effet, par le velouté, de donner envie de toucher les photos. Certes, les blancs ne sont pas parfaitement blancs, comme dans un tirage numérique, mais on a un relief, une vie, qui ont quelque chose de particulier.

Certaines des photographies sont toutefois tirées sur du papier argentique qui n'est pas du Cibachrome. D'ailleurs, il ne serait pas possible, en Cibachrome, d'obtenir un tirage aussi grand que celui d'Avenida parallel à Barcelone (fig. 4), parce que les lés de ce papier n'ont au maximum qu'une largeur de 1,27 m. Ou bien alors, il faudrait en juxtaposer deux, mais il y aurait une coupure au milieu de la photographie. Là, le format est de $1,80 \mathrm{~m} \times 2,35 \mathrm{~m}$.

\section{Grand Palais}

Avec le Grand Palais, vous restez dans le chantier, dans l'inachèvement. Et l'on retrouve ici la solidité des verticales et des horizontales. Les obliques n' informent en rien la profondeur, mais un X métallique trône au cour de l'image en barrant l'accès à l' espace du bâtiment (fig. 3). Le cadrage est osé.

Cette série a été réalisée en 2004 alors que le Grand Palais était en travaux. La trame constituée par les échafaudages de bois et les poutrelles métalliques permettait de compenser le côté narratif de l'image photographique.

Là aussi, on opère un décentrement en privilégiant les verticales et les horizontales. La composition est extrêmement précise, avec ce $\mathrm{X}$ métallique qui se trouve au milieu et la sédimentation des plans. Le choix de la frontalité permet, ici encore, de jouer sur l'ambiguïté de ce qui se trouve devant ou derrière. Il importait, pour que ce regard fonctionne, d'avoir une image totalement nette, sans effet de lumière, ni de perspective, ni de brume. Ainsi était prise à rebrousse-poil la neutralité de la photographie allemande : l'objectivité est respectée, mais elle concerne ici le fragment, non pas la photographie dans son entier. Cela produit un effet pictural singulier.

Avez-vous effectué des repérages préparatoires à la prise de vue?

À l'époque, le repérage n'était pas facile car il s'agissait d'un chantier. L'accès au site posait problème, on ne pouvait pas venir comme on le souhaitait. On ne savait pas si l'on serait autorisé à revenir le lendemain, si un boulon ne serait pas tombé entre-temps qui aurait rendu le site dangereux.

Il y a eu, certes, un travail de repérage qui m'a servi pour voir le lieu, la lumière, évaluer la manière dont je pourrais travailler. Mais les repérages plus précis, image par image, n'ont pas eu lieu. La suite s'est effectuée, comme dans les usines Renault, dans une certaine urgence. D'ailleurs, il n'y a jamais de repérage qui permette d'affirmer à coup sûr : je vais faire cette photo ! Un repérage n'est jamais qu'indicatif.

Parfois, j'effectue une première prise de vue. Il arrive que le point de vue ou la lumière ne conviennent pas. Si la photographie n'est pas bonne, je me dis que j'essaierai d'améliorer la fois suivante. Il y a donc une sorte de progression pas à pas qui laisse à penser que l'on va épuiser le sujet. 


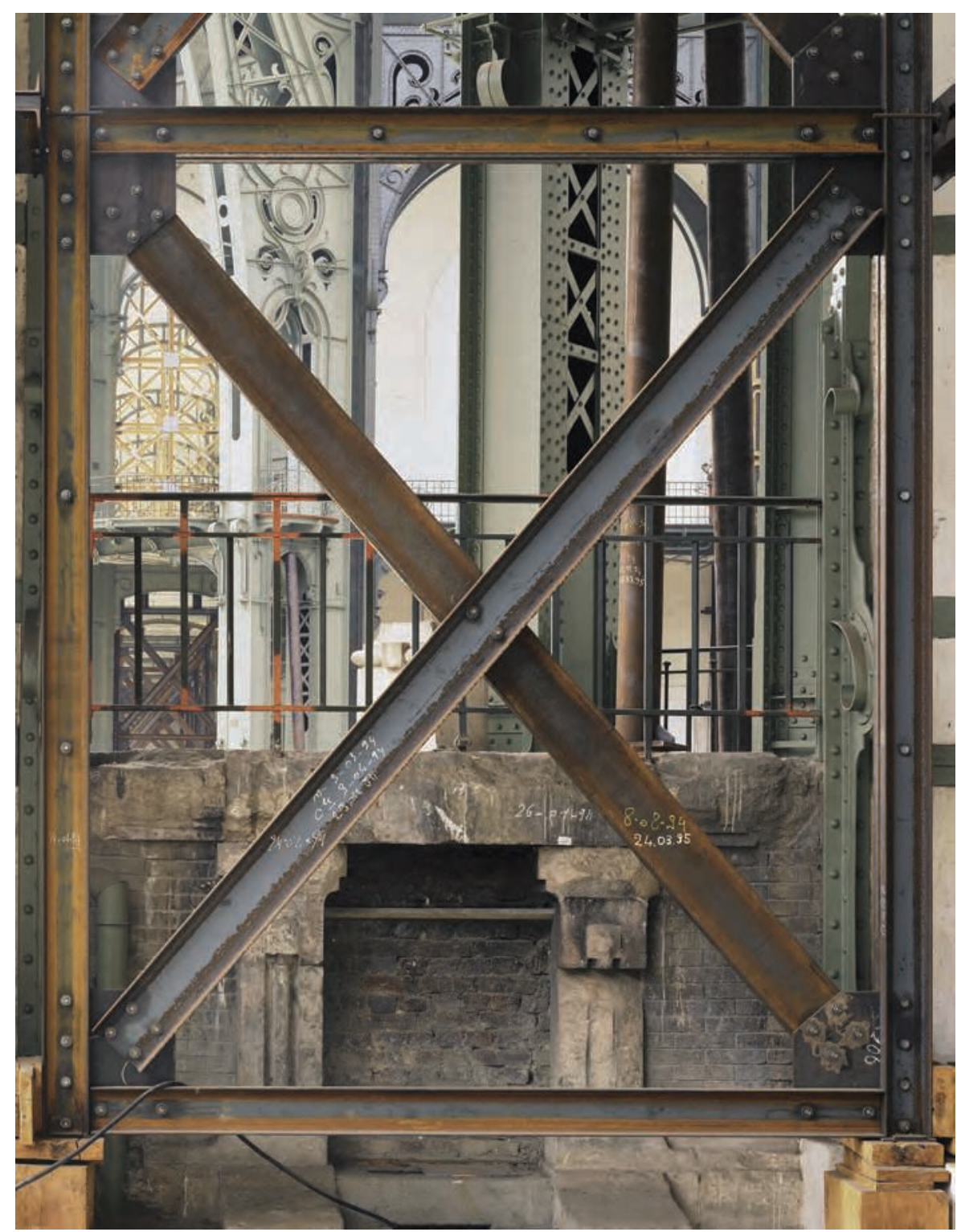

Fig. 3 : Grand Palais, 1997

(Ilfochrome, $157 \times 125 \mathrm{~cm}, 5$ exemplaires)

(c) Stéphane Couturier

À vrai dire, au sein du Grand Palais, les points de vue à retenir n'étaient pas nombreux. Pour photographier ces poutres et ce X, il n'y en a qu'un, pour moi. La grosse difficulté de ce genre de prise de vue est la profondeur de champ. Il faut à la fois trouver le point de vue convenable et obtenir la netteté sur l'ensemble des champs. Peut-être auraisje pu me placer plus loin mais alors il y avait une barre du chantier qui m'aurait gêné. Il faut à la fois avoir du recul et ne pas en avoir trop pour obtenir cette vision, pour être dans le fragment au moment de l'observation de l'œuvre.

\section{La réalisation des prises de vue est-elle rapide?}

Je n'ai jamais été un fou de technique et je ne suis pas très patient. J'ai donc envie de dire qu'il faut que les photos soient relativement simples à faire et intuitives. La plupart du temps, je suis seul, sans assistant. J'aime pouvoir rester, attendre la bonne lumière, revenir, mais rester libre de mes mouvements et, finalement, me dire : « Je vais attendre, mais peut-être que la photo ne se fera pas. » Et j'accepte cette idée que la photo ne se fasse pas, 
quand d'autres affirmeraient : «Je vais tout mettre en œuvre pour pouvoir la faire. Si la lumière est bonne, je vais en rajouter, installer des réflecteurs. » L'idée, pour moi, est de rester au plus proche de ce que l'œil peut voir, avec, de surcroît, la présence d'un cadre photographique.

En général, j'utilise des temps de pose assez longs, de quelques secondes, avec un diaphragme assez fermé. Les prises de vue sont réalisées lors de journées lumineuses mais sans soleil, en évitant les lumières fortes.

Sur cette photo du Grand Palais, il y a des inscriptions manuscrites, certainement techniques, effectuées à la craie sur les IPN, les poutrelles d'acier, peut-être par les responsables $d u$ chantier ou les ouvriers. Si l'image est vide, la présence humaine est là, comme elle l'est dans la mise en ouvre du chantier.

Ces inscriptions m'intéressaient. Quand le numérique a commencé à pointer le bout de son nez, les gens disaient : «Vous avez ajouté des inscriptions ! 》 Rapidement, avec l'arrivée des outils numériques, a surgi une forme de suspicion vis-à-vis des images. Mais l'ambiguïté m'intéresse et je préfère ne pas la lever : ces inscriptions étaient-elles effectivement présentes sur les poutres mêmes ou furent-elles ajoutées au tirage ? Que regarde-t-on ? De quoi s'agit-il ?

Le Grand Palais que j'ai photographié en 1997 est un grand corps de métal malade, un corps organique épaulé par une structure rigide de métal et de bois qui vient contrecarrer une architecture de volutes et de courbes.

Ces photographies réalisées à l'époque avec des procédés et des techniques de l'argentique préfigurent la mise en doute de la photographie. Elles annoncent la crise de l'analogique que le numérique engendrera incessamment.

\section{Barcelone}

Cette image (fig. 4) de l'Avenida Parallel à Barcelone est belle et étrange à la fois. Si nous la regardons de près, attentivement, nous voyons que se côtoient des fenêtres rectangulaires et des fenêtres arrondies, des balustrades de pierre et des balustrades de métal, des rideaux rayées rouge et jaune et des fenêtres sans rideaux. Quels ont été ici les processus de création? Le travail principal a-til été réalisé lors de la prise de vue ou, par la suite, en postproduction?

Cette photographie de l'Avenida Parallel à Barcelone a été réalisée en 2008-2009. L'idée était ici d'effectuer des prises de vue de deux immeubles distants de quelques centaines de mètres. L'un date du début du $\mathrm{xx}^{\mathrm{e}}$ siècle ; le second, des années soixante-dix. Ces deux images sont superposées fragment par fragment, si bien que ce que l'on voit est tantôt l'immeuble ancien, tantôt l'immeuble moderne.

Il s'agit ici de la série que j'ai intitulée Melting point, qui joue sur la superposition de deux images, véritable travail expérimental relatif aux nouveaux outils numériques. Je ne voulais pas rester dans les modalités de création des photographies que j'ai réalisées au Grand Palais en disant : "Le numérique, je ne veux pas y toucher ! »Certaines galeries avec lesquelles je travaillais pensent que l'argentique est préférable parce qu'ainsi, il n'y a pas de manipulations. Je ne suis pas d'accord avec cette argumentation. La manipulation n'est pas à rejeter comme quelque chose de sale. Une photographie argentique n'a pas les mêmes qualités qu'une photographie numérique, mais elle n'est à placer hiérarchiquement ni au-dessus ni au-dessous d'elle. La technique est un choix, elle est au service d'un point de vue, d'une recherche.

J'ai commencé à travailler en numérique au cours de l'année 2004. Plus exactement, j'associe la prise de vue argentique réalisée à la chambre avec le scanning des ektachromes, puis le travail sur ordinateur. Cette dernière étape consiste à superposer deux images l'une sur l'autre. La superposition de deux images n'est pas quelque chose de nouveau mais ici, les outils numériques permettent de choisir dans chaque image le fragment qui sera utilisé dans la superposition. Ainsi vais-je décider moi-même du dispositif de superposition et du rendu final. Pour chaque parcelle d'image, est-ce que j'utilise l'une des images ou les deux ? Est-ce que j'en utilise une à $10 \%$ et la seconde à $90 \%$ ou les fais-je apparaître à part égale ? Tantôt je fais apparaître l'une, tantôt l'autre. Cela marche très bien, même en laissant place à l'accident, au hasard.

J'ai matérialisé l'idée que l'on peut traiter la superposition pixel par pixel et choisir pour chacun d'eux 


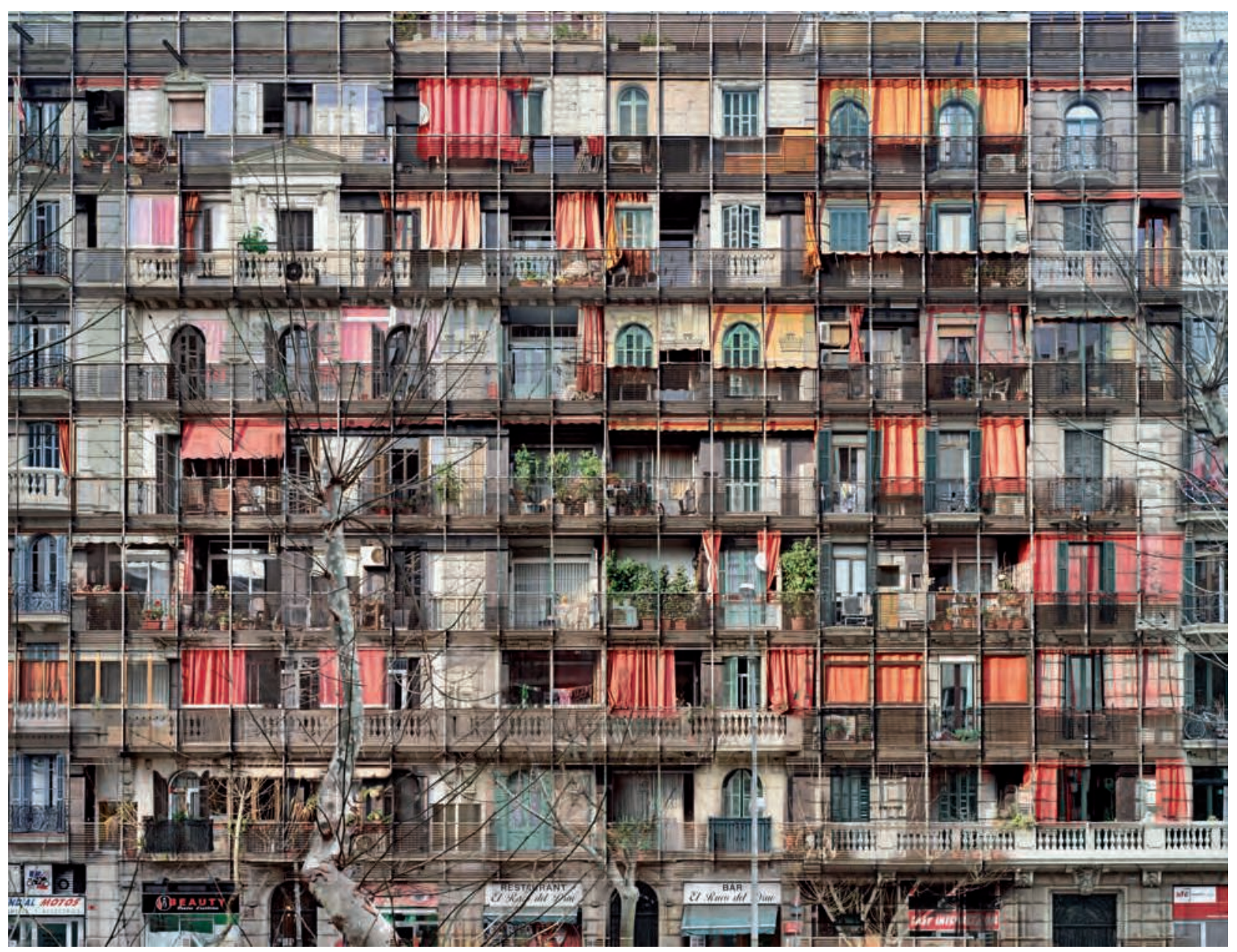

Fig. 4 : Barcelone, Avenida Parallel n 1, 2008-2009 (C-Print, $180 \times 235 \mathrm{~cm}, 5$ exemplaires) (C) Stéphane Couturier

la photographie que l'on fera apparaître. Travailler sur de grands formats incite à détailler l'image fragment par fragment. Là, le spectateur va découvrir - ou ne pas voir, d'ailleurs - un certain nombre de choses. L'idée, compte tenu du format, est de faire en sorte que chacun construise son parcours, découvre par lui-même des accidents, des éléments qui ne sont pas logiques. Ainsi, certains voient tout de suite qu'il y a superposition de deux immeubles : des balustrades sont en pierre, d'autres en fer forgé, cela n'est pas logique. Certains ne le voient pas. L'expérience de chacun fait que ce que l'on propose est mis en doute rapidement ou est accepté d'emblée. C'est cela qui est intéressant.

La qualité de ce regard dépend de la distance à laquelle se situe l'observateur. Si cette photographie est observée à cinq ou dix mètres, la superposition n'est pas visible. Mais lorsqu'on s'approche, on commence à voir des choses bizarres.
Ce travail de superposition est toujours effectué à partir de deux images seulement. Certains auteurs superposent trois, cinq, dix, vingt ou même plusieurs centaines d'images. Pourquoi pas ? Mais chaque fois, ces superpositions de la série Melting point ont été réalisées à partir de deux images.

Certes, il s'agit d'utiliser les outils numériques, mais avez-vous souhaité marquer la différence d'écriture photographique avec les séries précédentes ou, à l'inverse, insister sur une continuité, et cela malgré les ruptures technologiques?

Dans un sens, le vocabulaire utilisé est le même que pour les photographies du Grand Palais. C'est-à-dire la frontalité de la prise de vue, l'intérêt porté au fragment, le travail de composition à l'intérieur du cadre photo avec une déhiérarchisation des sujets. Chaque objet de 
l'image a la même valeur, est porté sur le même plan que les autres. Il n'y a plus de proche ou de lointain, pas d'obliques de perspective, pas de perspective atmosphérique... Ainsi, nous ne nous trouvons pas dans un "ça a été », comme le disait Roland Barthes, même si, en un sens, il y a eu présence au moment de la prise de vue. L'instant décisif, au sens où l'entend Henri CartierBresson, existe car il a bien fallu faire des photos à un moment donné, avec un protocole précis, mais l'instantanéité de la prise de vue n'est pas recherchée ; elle ne signe pas ici le talent du photographe. À l'opposé, l'idée est de remettre en cause les fondamentaux de la photographie artistique. Cette façade pourrait bien continuer à l'infini, ne jamais s'arrêter. Nous sommes dans le mouvement, dans une lecture dynamique.

Nous jouons avec quelque chose qui ressemble à la persistance rétinienne. Regarder un élément de l'image, puis, quelques secondes plus tard, un second élément conduit à les superposer, les confondre, opérer une continuité qui, en réalité, n'existe pas. Il ne s'agit pas de faire une image de synthèse mais une image hybride qui montre l'hétérogénéité d'une avenue de Barcelone tout en jouant sur deux temporalités.

Mais entre le travail que j'avais effectué au Grand Palais et celui-ci, partie prenante de la série Melting point, les pratiques ont changé. Au Grand Palais, la question du tirage restait importante. Ici, avec Avenida Parallel, le travail de postproduction est beaucoup plus important. Avant d'effectuer un tirage papier à l'aide d'une imprimante numérique, les ektas ${ }^{2}$ sont scannés. Dès lors, il est possible de mémoriser la chromie pour refaire un second tirage qui soit très proche. Il ne sera cependant jamais exactement semblable à l'image initiale, car la chimie du tirage, le choix de papiers qui possèdent leurs propres caractéristiques, jouent beaucoup, mais on sera plus proche d'un précédent tirage qu'avec les procédés argentiques.

J'ai travaillé sur les superpositions d'images à partir de 2004. Aujourd'hui, je recommence à travailler en Cibachrome sans forcément faire des superpositions d'images. Cette superposition, je l'ai pratiquée, mais je ne dis pas que c'est terminé. Il s'agissait aussi, à un moment donné, d'apporter une réponse à une nouvelle technologie et de voir simultanément comment l'on pourrait trouver des solutions.
Il est intéressant de voir que la manière dont les spectateurs regardent une image a évolué durant ces dernières années. Ils veulent aujourd'hui des photographies spectaculaires, plus colorées, de format plus grand. Personnellement, j'ai toujours choisi des formats de grande taille en ayant l'idée de faciliter l'entrée à l'intérieur même de l'image, de faire en sorte que l'on puisse s'approcher de très près. Pour cette raison, je travaillais avec des chambres photographiques qui facilitaient les grands agrandissements, plutôt qu'en $24 \times 36$.

\section{San Diego}

Cette photographie (fig. 5) est devenue célèbre. Elle nous semble étrange tout en étant documentaire, à tel point que l'on s'interroge : quelle est la part de la réalité, quelle est celle de l'artiste?

On est en Californie, dans la banlieue de San Diego, au tout début des années deux mille. Il y a toujours cette frontalité, la même fenêtre. D'une certaine manière la composition est voisine de celle de la photographie du Grand Palais. Mais on est ailleurs, dans un autre monde avec cependant, toujours, la dimension d'un Work in progress, d'un chantier.

J'ai voulu photographier cette ville qui se construit, sans fin... s'étend, empiète sur le désert. Nous sommes à l'intérieur même d'une maison en construction, dominant d'autres maisons par une ouverture dans l'ossature de bois. Celle-ci est légère, souple, afin de prévenir les effets des tremblements de terre.

On reconnaît le petit gazon, le garage, les voitures, les baraques de chantier et même une cheminée de climatisation. Il n'est pas question de magnifier ou de dénoncer ce genre de paysage. Ici, la composition ménage un peu de ciel : il s'agit de l'exception qui confirme la règle. L'idée est bien toujours que l'œil se promène dans

2. Ektachrome (sous-marque de Kodak) correspond à une gamme de films photographiques inversibles, disponibles en plusieurs formats, notamment en grands formats. 


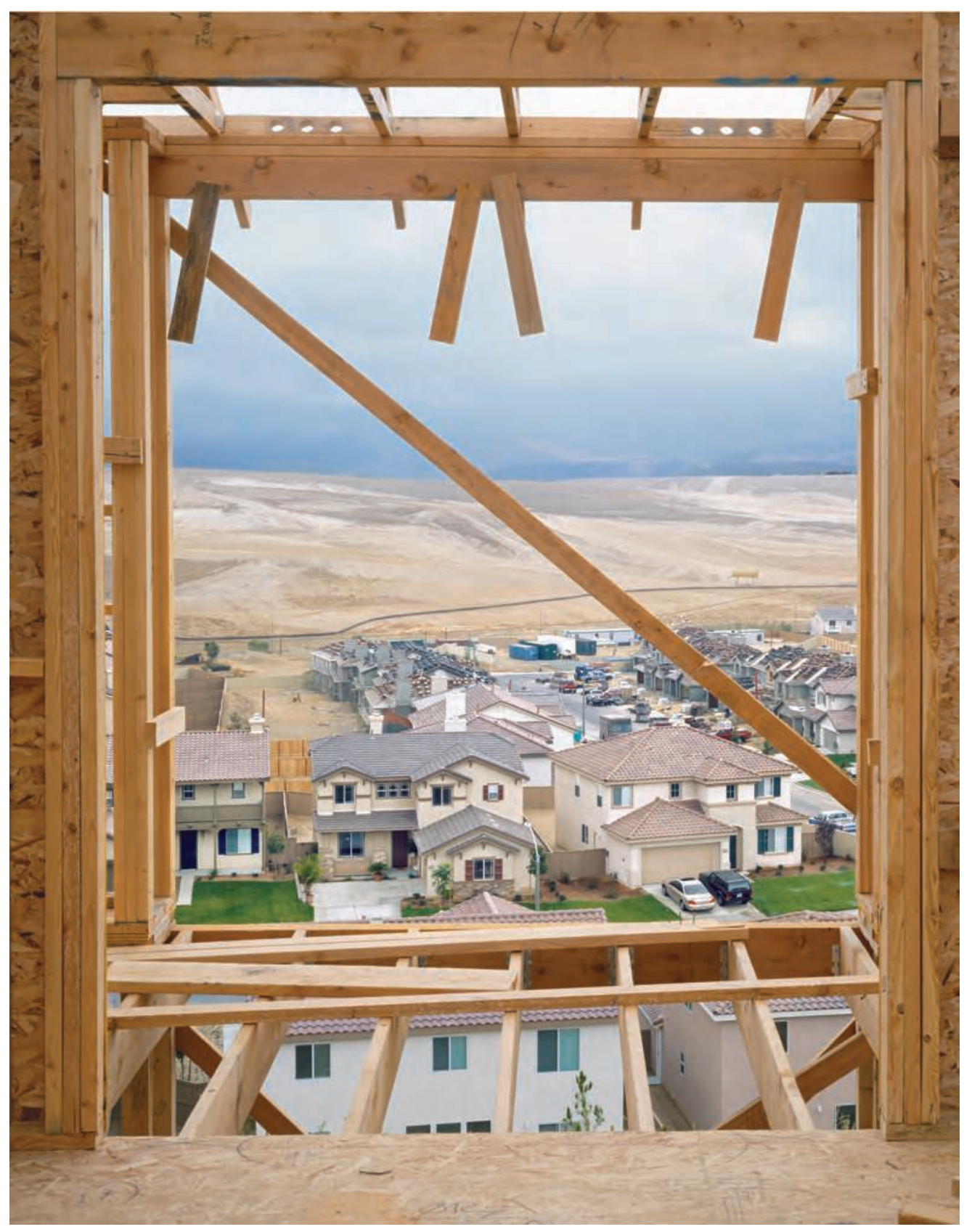

Fig. 5 : Série Landscaping, San Diego, fenêtre, East lake greens, 2002 (Ilfoflex, $200 \times 158 \mathrm{~cm}, 8$ exemplaires)

$$
\text { (c) Stéphane Couturier }
$$

les différentes parties de l'image alors que l'on reste dans la photographie documentaire. On est à San Diego, on pourrait être ailleurs; reste cependant bien ancrée cette idée de travailler sur les transformations du paysage construit.

La difficulté vient du fait que, par le projet même, on se trouve dans des lieux en mutation. Si j'étais venu une semaine ou dix jours auparavant, je n'aurais pas capté cette fenêtre telle qu'elle était là, au moment de la prise de vue. Le concept de chantier exclut, de fait, la notion de repérage : ce que l'on aura à repérer sera différent le lendemain. Nous sommes confrontés en permanence à quelque chose de nouveau. 
Qu' est-ce qu' une telle photographie nous apprend sur les démarches de son auteur?

Ce n'est pas à moi de répondre, mais vous remarquerez qu'il y a toujours une recherche de frontalité, un travail sur les lumières. À San Diego, il n'y a guère que quinze jours à trois semaines par an de journées non ensoleillées. Encore ne sont-elles pas toutes favorables : il peut pleuvoir, une tornade peut surgir... Donc, pour obtenir une image sans ombres comme celle-ci, c'est extrêmement compliqué. En effet, l'ektachrome ne tolère pas plus de trois-quatre diaphragmes de différence au sein d'une même image. Il oblige à l'obtention d'une lumière très homogène. Je n'ai jamais rien éclairé : toutes les photographies sont réalisées en lumière naturelle. Ici, finalement, c'est la météo qui a déclenché la prise de vue. Il n'y avait pas de repérage possible. Je suis arrivé là... En un sens, il s'agit presque d'un instant décisif.

Je suis arrivé un samedi matin. Il y avait un problème d'accès : il s'agit d'un chantier où donc, $a$ priori, il est interdit d'entrer, mais j'ai pu y accéder. J'étais au premier étage d'une maison en construction. Ce qui est étonnant, c'est que cette photo a été, pour moi, très facile à réaliser. Elle s'est donnée très rapidement, j'ai pu la faire en quelques minutes. C'était si rapide que tout d'abord elle ne m'a pas intéressé. Je ne l'ai pas choisie, je l'ai mise de côté. Elle me semblait trop emblématique : j'y voyais des constructions industrielles dans le désert qui donnaient naissance à des jouets, à des petites voitures. Ce n'est qu'au bout d'un an et demi ou deux qu'elle a resurgi, qu'elle s'est imposée. Je me suis posé la question : mais pourquoi ? Je n'ai toujours pas de réponse satisfaisante.

On pouvait se demander : dans quelle ville, dans quel pays sommes-nous ? En Israël, au Maroc, en Espagne, en Asie, en Amérique latine ? On retrouve partout le même type de constructions, comme un clonage du paysage. D'ailleurs, la série s'intitule Landscaping, ce qui signifie « Transformation du paysage ». Je me situais ainsi au carrefour de différentes disciplines ; j'étais à la fois dans la photographie, dans l'art contemporain, dans la politique urbaine, dans l'architecture.

Travaillez-vous encore sur commande, avec des architectes ou des paysagistes?
Je suis autodidacte. Pire, j'ai tendance à ne pas aller vers quelqu'un qui va me dire qu'il va falloir photographier en fonction de l'histoire de l'urbanisme, de celle de l'architecture. J'essaie pourtant de me documenter afin de ne pas rater quelque chose d'important, mais j'aime aussi tirer parti du hasard.

\section{Chandigarh}

Ces deux photographies (fig. 6 et 7) bénéficient de riches coloris. Le premier de ces deux bâtiments fut construit par Le Corbusier, le second "à la manière de Le Corbusier ». Si les coloris chatoyants et l'architecture créent d'indiscutables liens entre ces deux photos, de nouveau la même ambiguïté se manifeste : qu'est-ce qui appartient à la réalité ? Qu'est-ce qui relève des dispositifs de prise de vue ou mis en cuvre lors de la postproduction?

Lahore, qui fut jadis la capitale du Penjab, se trouve aujourd'hui au Pakistan. Chandigarh, capitale des deux états, le Penjab et l'Aryana, est une ville nouvelle dont le premier architecte fut Albert Mayer. Mais en 1951 Nehru fait appel à Le Corbusier, qui travaille non seulement comme architecte mais également comme artiste. Dans cette ville se trouve la plus forte concentration d'œuvres de Le Corbusier au monde.

La première photographie (fig. 6) résulte d'un travail de superposition de deux réalités : une architecture et une tapisserie monumentale de Le Corbusier réalisée à partir de son travail architectural. Ici, la superposition est pensée dès la prise de vue. J'ai agi comme un joueur de cartes qui réunirait des familles. Après les prises de vue est venue la seconde étape du processus : le jeu de superposition et la mise en forme d'œuvres graphiques.

La seconde photographie (fig. 7), quant à elle, nous conduit dans le postcorbusien. On n'est plus du tout dans Le Corbusier. Ces décors ne sont pas le fait de Le Corbusier mais d'artistes indiens qui ont œuvré à la manière de Le Corbusier. La photographie est un clin d'œil car on se trouve ici dans les faubourgs de Chandigarh. Le travail de superposition montre les traces des tapisseries qui sont à l'intérieur du bâtiment. 


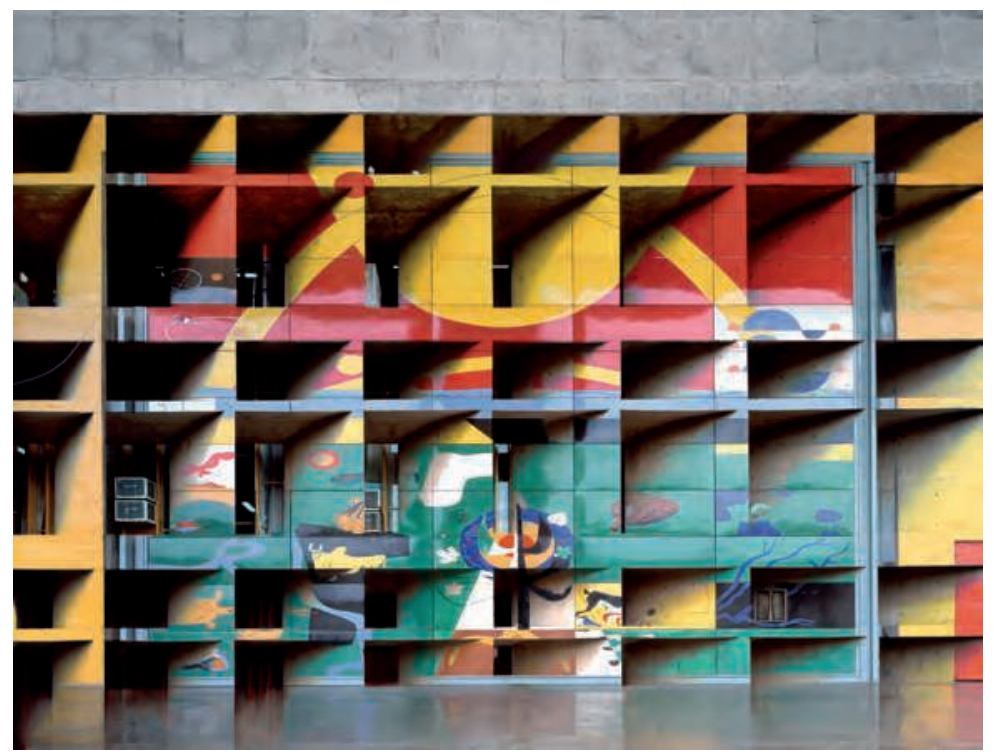

Fig. 6 : Chandigarh Replay, Chandigarh, Assemblée n 1, 2006-2007 (C-print sur Diasec, $160 \times 200$ cm, 5 exemplaires) (c) Stéphane Couturier

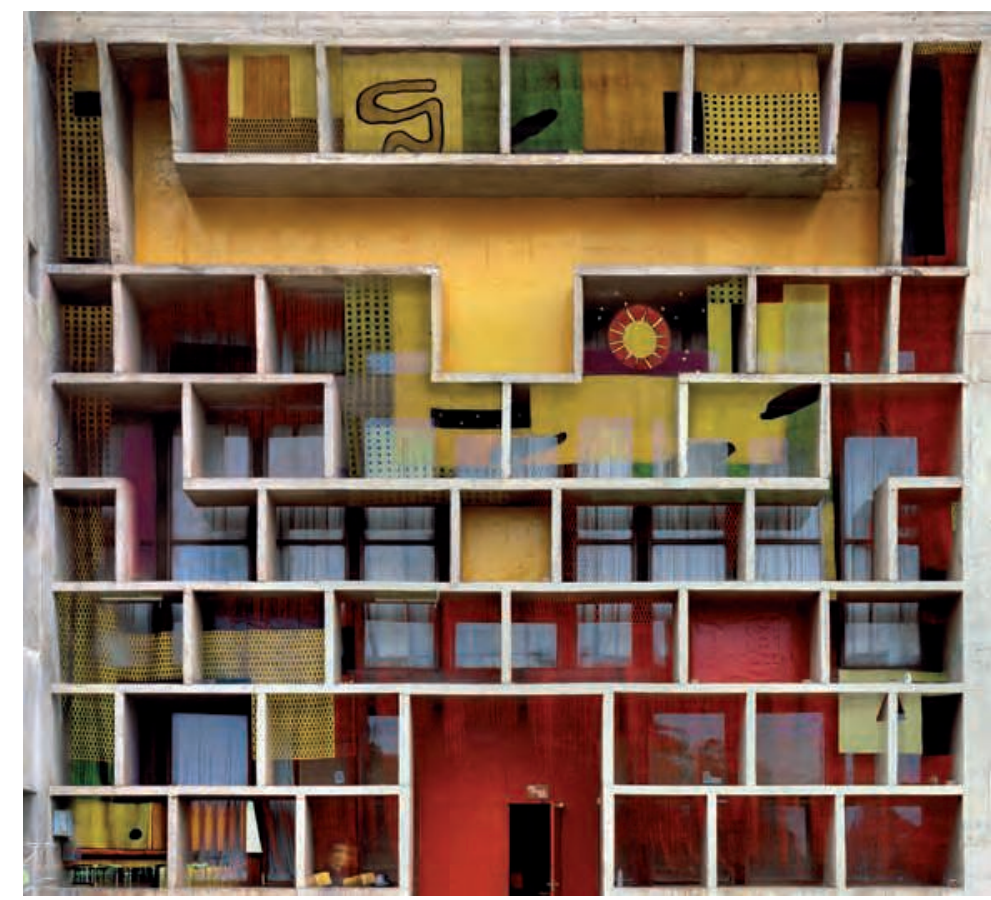

Fig. 7 : Chandigarh Replay, Haute cour de justice, 2007 . Résultat de la superposition des ektas 1 et 2 (C-print sur Diasec, $180 \times 198 \mathrm{~cm}$ ) (c) Stéphane Couturier

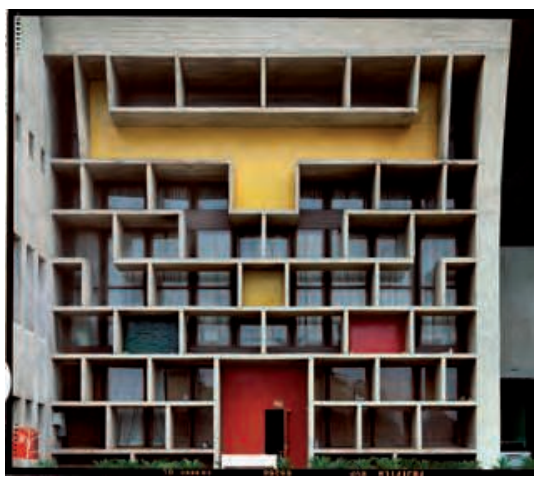

Ekta $^{\circ} 1$

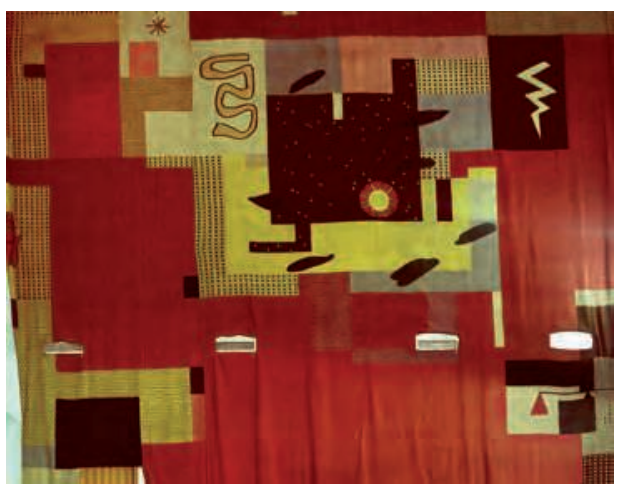

Ekta $n^{\circ} 2$ 


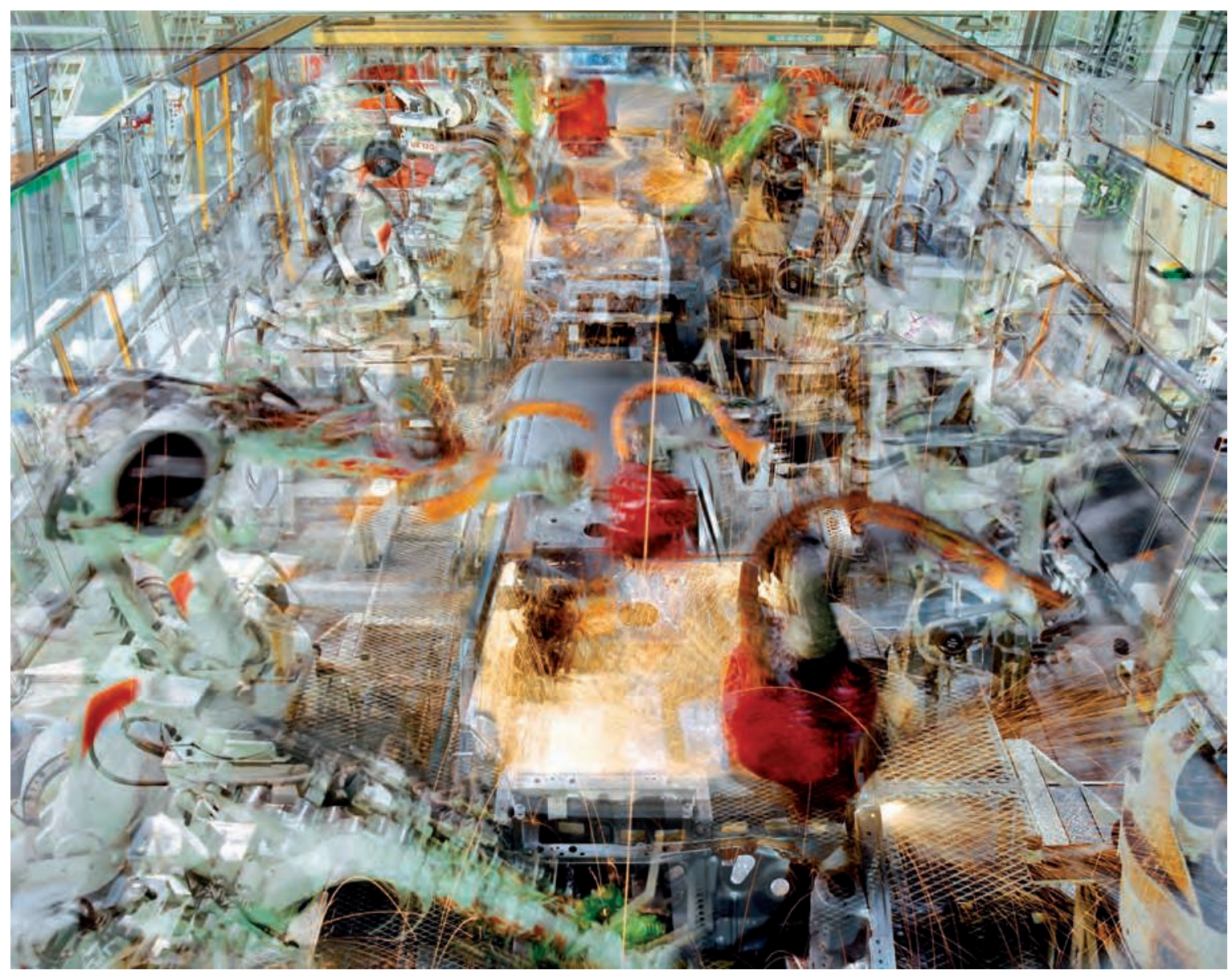

Fig. 8 : Toyota, Valenciennes, 2005

(C-Print, $160 \times 212 \mathrm{~cm}, 5$ exemplaires)

(c) Stéphane Couturier

\section{Toyota}

Ici (fig. 8), l'image vibre, tant par les formes que par les couleurs. Nous ressentons en nous-mêmes ces vibrations. $D$ 'où provient cet effet? Comment fut-il obtenu et quels sens leur auteur lui confère-t-il?

Voici l'une des photographies de la série Melting Point réalisée au sein de l'usine Toyota de Valenciennes. Ici aussi la superposition a été effectuée lors de la prise de vue. Ce que j'ai trouvé intéressant, c'est qu'avec la superposition des images, j'étais plus proche de ce que j'avais ressenti à l'intérieur de l'usine que si je n'avais réalisé qu'une seule photographie réaliste. La superposition était plus sonore, plus vibrante, plus vivante, plus mouvante. Rendant mieux compte du bruit, mais aussi de la complexité du lieu, de sa sophistication, de son mystère, elle se rapprochait plus de l'effet que je souhaitais.
Toutes les photographies que j'ai réalisées en 2005 dans l'usine Toyota sont des images de grand format et obéissent à ce principe de superposition. Là, on devine un ouvrier. La superposition l'a un peu gommé. De temps en temps, dans cette série Toyota, il y a des personnages. On les devine. Le plus souvent, cependant, ils sont absents. Ainsi la photographie ne se limite-t-elle pas à une simple image : elle inclut la bande-son et plus encore l'effet kinesthésique. On vit l'intérieur de l'usine.

En contrepartie, l'idée est de conserver les couleurs de la réalité. Il n'y a pas, ici, d'intervention. Le rouge est le vrai rouge. Les protections étaient roses. De temps en temps, lorsque deux couleurs sont additionnées, on obtient des formes un peu violacées. Mais l'idée est de protéger, de retenir ces phénomènes de sursaturation pour aboutir à des photographies qui soient fluides et hybrides. Nous sommes dans la photographie mais celleci tend vers la vidéo. Il s'agit de processus discontinus : la seconde image introduit dans la première quelque 


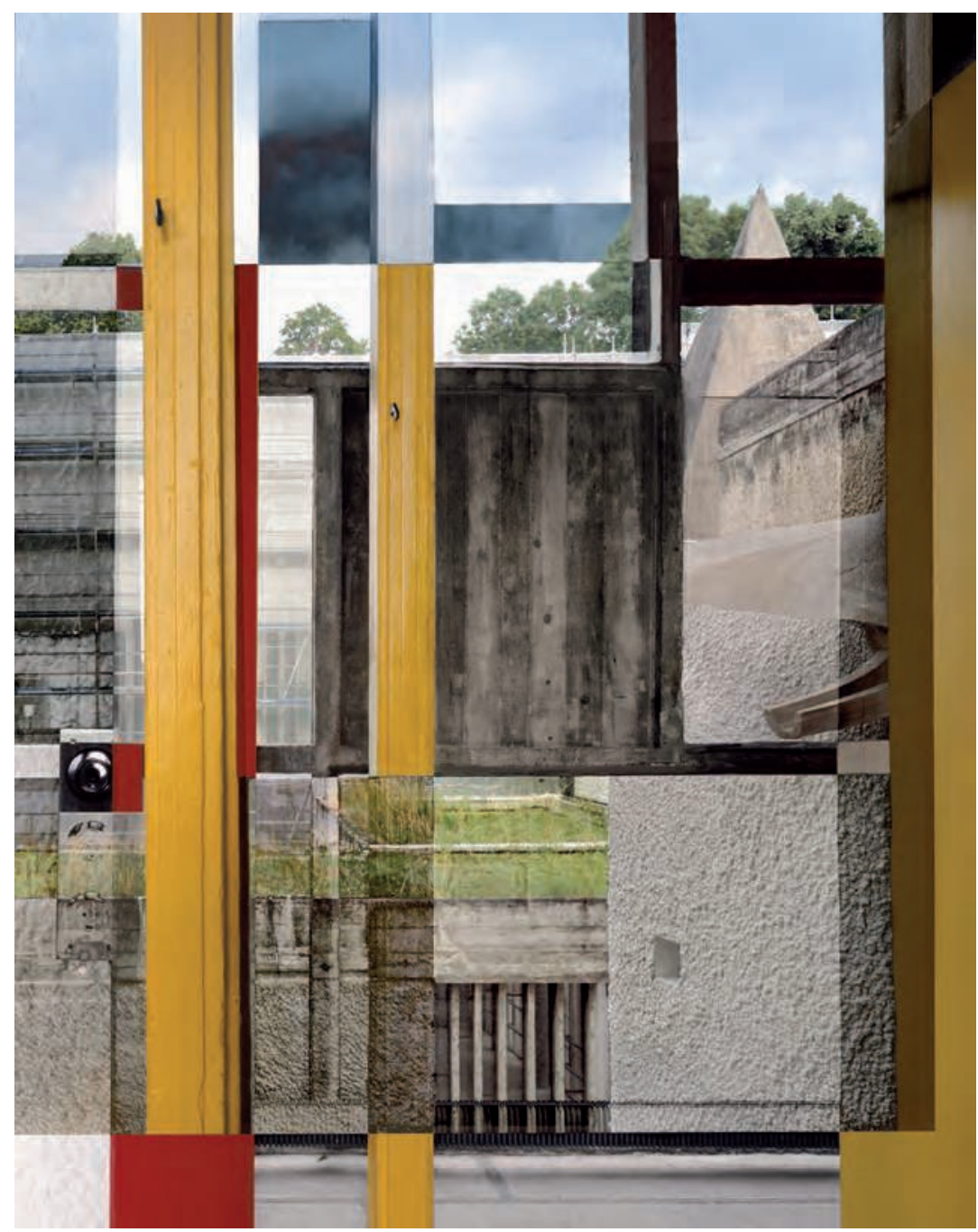

Fig. 9 : Série Melting point - Couvent de La Tourette, photo n 1, Commune d'Eveux, 2009-2010 (C-Print, $130 \times 103 \mathrm{~cm}, 5$ exemplaires)

(c) Stéphane Couturier

chose qui la fait bouger. Je travaille peu à peu, sur ce terrain, entre photographie et vidéo. Je réfléchis à leurs frontières : à quel moment est-on dans la photographie ? à quel moment se trouve-t-on dans la vidéo ?

Ces images de l'usine Toyota de Valenciennes font donc naître toute une interrogation sur l'image fluide, non statique mais qui, cependant, est riche d'un enracinement documentaire. À l'époque, l'usine Toyota fabriquait des Corolla, pas des Yaris : nous ne sommes pas dans l'abstraction.

\section{La Tourette}

Dans cette photographie (fig. 9), le fait est rare, un morceau de ciel apparaît. L'ensemble, d'une géométrie aussi rigoureuse qu' un tableau de Piet Mondrian conserve certainement un ancrage documentaire. Les dispositifs de prise de vue sont-ils, ici, différents des précédents?

Ici, nous rejoignons Le Corbusier dans l'église du couvent de La Tourette, près de Lyon. Il s'agit d'une superposition née d'un travail de prise de vue en champcontrechamp réalisé depuis l'intérieur de l'église. On devine l'autel, mais on aperçoit aussi les arbres et le ciel. Il y a tout un travail de façonnement avec des masses, des rectangles, des carrés.

La chambre photographique est l'outil idéal pour réaliser de véritables compositions, à partir d'un élément central. En permettant de voir précisément sur l'écran dépoli la scène photographiée, en aidant précisément aux repérages, elle favorise l'agencement de rectangles, 
de carrés, d'à-plats. Ces éléments sont ainsi sortis du contexte et, tout à coup, on obtient des formes nouvelles, qui vont au-delà de l'objet représenté. Lorsque l'on s'attache au fragment, on est dans l'abstraction. C'est cela qui m'intéresse : passer du macro au micro, effectuer des allers et retours pour, finalement, s'interroger : où se trouve-t-on ? De quoi s'agit-il ? Qu'est-ce que je regarde ? J'aime que le spectateur reste dans l'ambiguïté, qu'il s'intéresse à la scène photographiée tout en étant dans la composition. Faire une photographie de côté, cela ne m'intéresse pas. Pour moi, la frontalité est une véritable signature : elle met en scène des plans, brouille les repères classiques de la profondeur, déhiérarchise. On ne sait plus ce qui est devant, ce qui est derrière. Il s'agit d'une forme de théâtralisation, une mise en scène. Je pars des trois dimensions pour avoir un effet d'à-plat. Les photographies, en général, sont conçues pour donner du relief : la lumière y joue un rôle fondamental. Mais moi, je fais l'inverse, j'utilise des éléments pour créer une sorte de platitude. Lee Friedlander m'a beaucoup influencé.

Aujourd'hui, je suis en train de revenir au Cibachrome. Après avoir gesticulé avec Photoshop, j'ai envie de revenir à des choses simples. La composition de l'image est importante, mais aussi la qualité du tirage.

Le problème est que les galeries utilisent aujourd'hui des tirages numériques qui se valent tous, qui sont tous réalisés de la même manière et cela devient très ennuyeux. Nous arrivons à une époque où l'uniformité née de la multiplication des tirages numériques rend l'imperfection du Cibachrome intéressante. Avec les tirages numériques, il n'y a pas de surprise : le blanc va être blanc, le rouge va être rouge. Avec le Cibachrome, le blanc n'est jamais blanc. Et là, cela devient intéressant, à un moment donné, de sentir la part du tirage, sa lourdeur ; sa pesanteur. Le problème est que rares sont les personnes qui parlent de cela. Aujourd'hui, il arrive souvent, hélas, que l'on juge d'une image à partir d'un écran. Mais alors, on juge la composition, pas la qualité des blancs. Il faut nourrir les blancs. Il y a trente-six mille sortes de blancs mais les photographes, souvent, oublient cela. Ils ne font plus guère attention au tirage car il existe aujourd'hui des systèmes d'imprimantes perfectionnés avec de nombreuses couleurs.

Je suis, personnellement, moins intéressé par le sujet représenté que par la manière de le traiter. La qualité du rendu, c'est ce qui fait qu'une image peut être d'une banalité à pleurer ou, à l'inverse, d'une belle sensualité.

Quand le numérique est arrivé, les gens disaient de mes photographies : «C'est du numérique ! » J'étais pris à mon propre piège en m'exclamant : «Non, ce n'est pas cela ! »; j'avais l'air de dire que l'argentique était mieux que le numérique. Et pourtant, je voyais le moment où il fallait réagir : «Attendez, je peux faire aussi autre chose, je vais expérimenter ces nouveaux outils !»

Aujourd'hui, je fais des vidéos à partir de photographies afin de leur donner encore plus de fluidité, de continuité. Je travaille avec la vidéo, sans que cela m'empêche de revenir au Cibachrome. C'est cela qui est intéressant : avoir un pied dans les technologies d'aujourd'hui tout en sachant de quoi l'on parle lorsque l'on fait un tirage. J'aime m'interroger : «Qu'est-ce qu'un blanc ? »'aime sortir un tirage Cibachrome tel que j'ai envie de le sortir, parce qu'à un moment donné cela permet de réfléchir sur ce qu'est, aujourd'hui, une œuvre photographique.

\section{La fin annoncée des tirages Cibachrome vous inquiète- t-elle?}

La fin du Cibachrome ne m'inquiète pas vraiment. Elle signe la fin d'une époque, voilà tout.

\begin{abstract}
Alstom
Dans ces deux photographies (fig. 10 et 11), la lumière est dorée. D'où vient cette couleur puisque vous n'avez pas éclairé de manière artificielle?

Là, on se trouve chez Alstom. Il m'a fallu six mois ou un an pour finaliser le travail. L'ektachrome crée une lumière dorée. Si j'avais réalisé cette photo en numérique, les couleurs n'auraient pas été les mêmes car le numérique aurait d'emblée corrigé les dominantes. Or, il y a des éclairages au iodure de sodium qui créent ce côté doré sur le métal et là, tout à coup, cela devient intéressant.

Il est en effet intéressant de constater que la redécouverte de certaines choses par l'imperfection de l'argentique permet d'avancer dans l'utilisation du numérique.
\end{abstract}




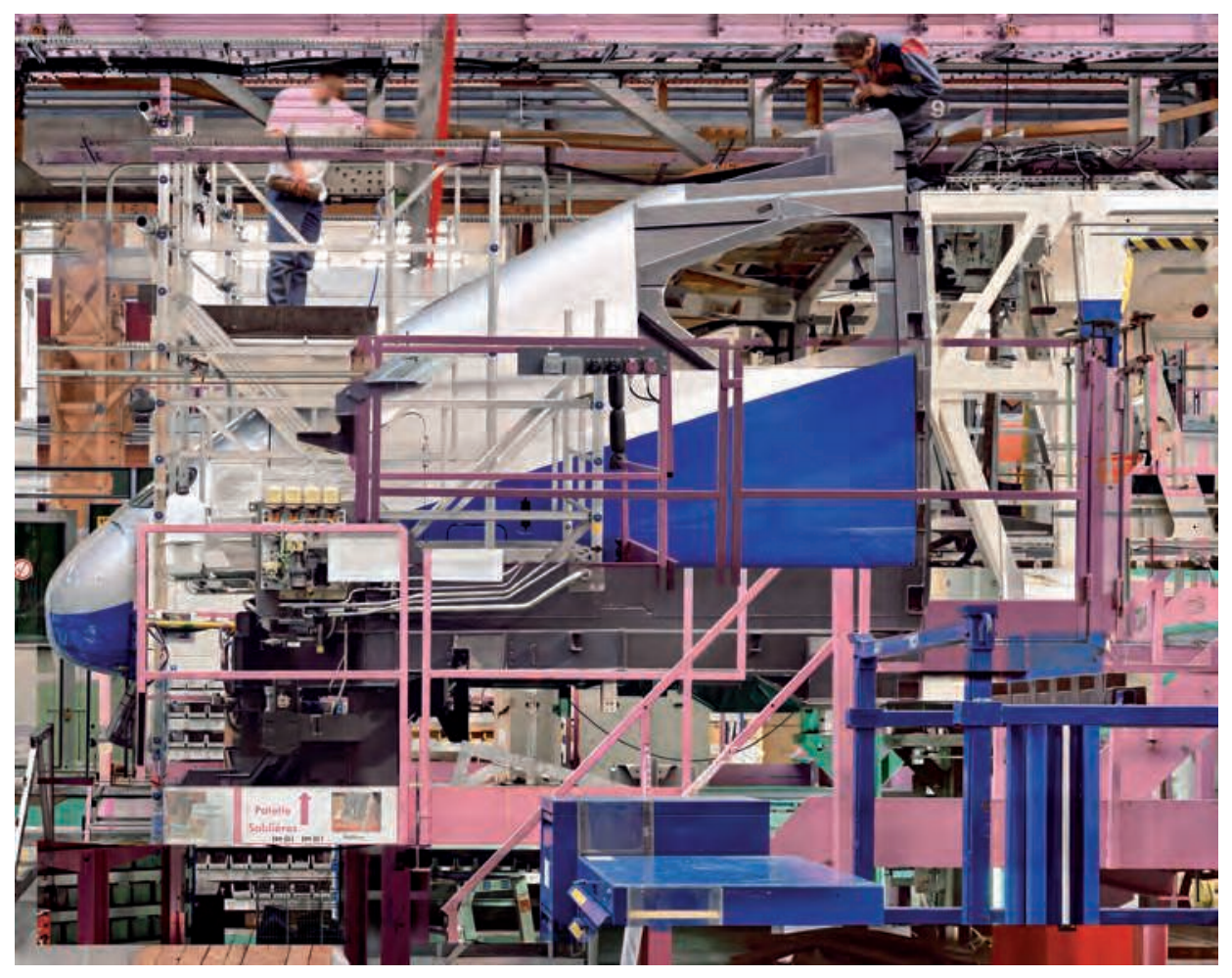

Fig. 10 : Alstom Belfort, 2009

(C-print, $180 \times 230 \mathrm{~cm}, 5$ exemplaires)

(c) Stéphane Couturier

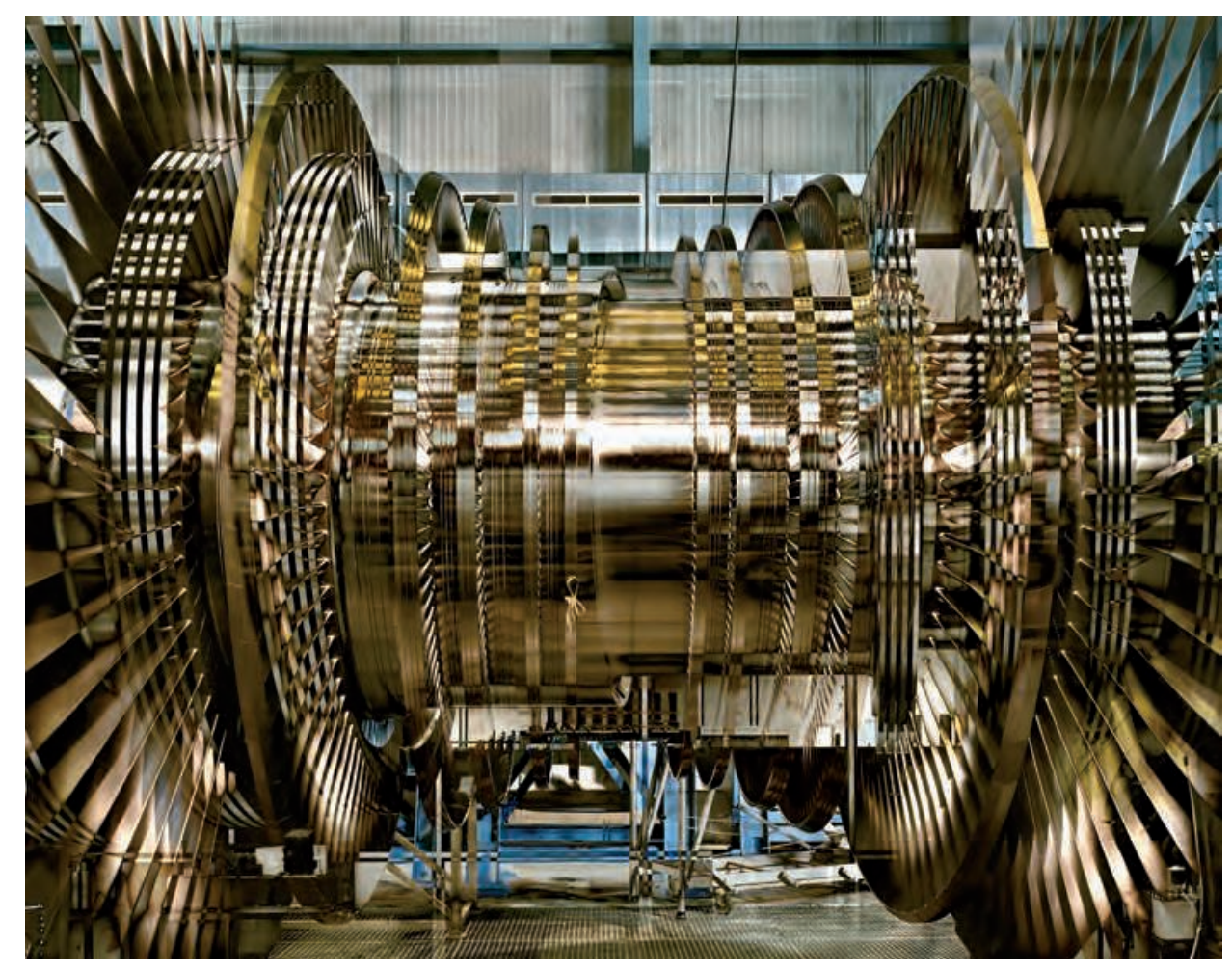

Fig. 11 : Alstom Belfort, 2009-2012, photo ${ }^{\circ} 16$, Halle Power (C-print, $160 \times 200 \mathrm{~cm}, 5$ exemplaires) 


\section{Villa Noailles II}

Par exemple, j'ai réalisé dans la villa Noailles, à Hyères, des photographies jaunes que j'ai tirées en Cibachrome (fig. 12). Je n'ai jamais pu les tirer en numérique : je ne parvenais pas à retrouver la sensualité du jaune doré. Les tirages numériques sont décevants, mais les technologies peuvent évoluer, bien sûr. Aujourd'hui, le numérique tend à singer l'argentique. On recherche le grain de l'argentique en utilisant des logiciels qui vieillissent les images pour leur conférer un aspect vintage. Malheureusement, dans les laboratoires, de plus en plus rares sont les personnes qui savent lire la chromie. Certains photographes acceptent ainsi des tirages de mauvaise qualité. On peut être découragé par le fait que très rares sont ceux qui perçoivent la différence entre un bon et un mauvais tirage. Il est difficile de maintenir la rigueur de la qualité quand $99 \%$ des gens ne le voient pas. Certains photographes commettent l'erreur d'estimer que ce $1 \%$ de ceux qui voient est négligeable alors que, justement, c'est la qualité même du tirage qui fait passer d'une photographie intéressante à une image qui a ce petit quelque chose en plus qui la fait sortir du lot pour devenir immortelle.

Il arrive qu'un tirage m'étonne moi-même. C'est une joie ! Mais souvent, les photographes ne cherchent plus ce plaisir du tirage. Ils conservent le plaisir de la prise de vue, pas celui du tirage. Personnellement, j'ai toujours été très interventionniste vis-à-vis des tireurs. Même avec des professionnels de qualité, comme Choi ou Roland Dufau, je fais refaire les tirages jusqu'à ce que je sois content et cela, bien qu'ils sachent parfaitement ce que je souhaite. Il m'arrive de faire refaire le travail jusqu'à cinq fois. Mais alors, les tireurs ne sont pas contents, parce que le papier Cibachrome, la chimie du tirage, les locaux, sont coûteux. Il est intéressant de constater que les personnes qui font effectuer leurs tirages Cibachrome par Choi sont moins photographes qu'artistes. Ceci pour dire que ces personnes n'ont pas pour préoccupation le sujet représenté mais bien la matière même de la photographie.

Le problème reste celui de la figuration. La question qui revient en permanence est celle-ci : «Qu'est-ce que cela représente ? » Les séries Melting Point visent à casser cette narration. Mais même s'il y a abstraction, il $\mathrm{y}$ aura toujours figuration parce que l'on voudra toujours retrouver « ce que cela représente ».
Je suis très lent, mais au moment où une photo se présente ou même quelque chose qui a besoin de se faire, je suis rapide. Je suis rapide lorsqu'il y a urgence. Je suis un photographe de terrain. Je ne suis pas dans mon studio, je suis à Séoul, à Pékin, à San Diego... ou ailleurs. Sur le terrain, je suis toléré mais dès l'instant suivant, je peux devenir indésirable. Cela n'est pas dû au choix de l'appareil de prise de vue. C'est paradoxal, mais plus il est gros, plus les gens se disent : "S'il est là, c'est qu'il a une autorisation ! » Avec la chambre, à la limite, vous passez inaperçu, quand un petit appareil photo aurait échoué à vous rendre invisible. Avec la chambre, on est tellement visible qu'on finit par être oublié. Alors, les gens regardent : dès lors, nous faisons partie des meubles, du paysage urbain. Je reste toujours intéressé par la manière dont les gens regardent une photographie. J'aime bien, lorsqu'il y a une exposition, les écouter, incognito. Cela implique une certaine humilité... Mais lorsque quelqu'un regarde une image, ce qui m'intéresse, c'est par quoi il commence et par quoi il finit. J'aime tous ces petits détails, ce mélange de surfaces achevées et d'un chantier qui est ni fait, ni à faire.

\section{Les archives}

Comment et sous quelle forme conservez-vous vos archives photographiques? Cette question vous pose-telle problème?

Je conserve tous les ektachromes, mais je me débarrasse des tirages ratés, du moins je n'en conserve que très peu. Je n'ai pas la place nécessaire. Ces ektachromes sont les positifs à partir desquels seront effectués les tirages Cibachrome. Ils en constituent la matrice. Au sein de ces ektas, je distingue les premiers choix et les seconds choix. Autrefois, lors du tirage, on utilisait des masques de contraste afin de doser la lumière qui atteignait telle ou telle partie de l'image. Il fallait ternir ces masques de cette manière-là. Aujourd'hui, ces masques sont devenus inutiles car on scanne les ektas.

Les ektas sont conservés dans des boîtes. Je les regarde de temps à autre. Malheureusement, je ne gère ni la température, ni le degré hygrométrique de leur conservation. 


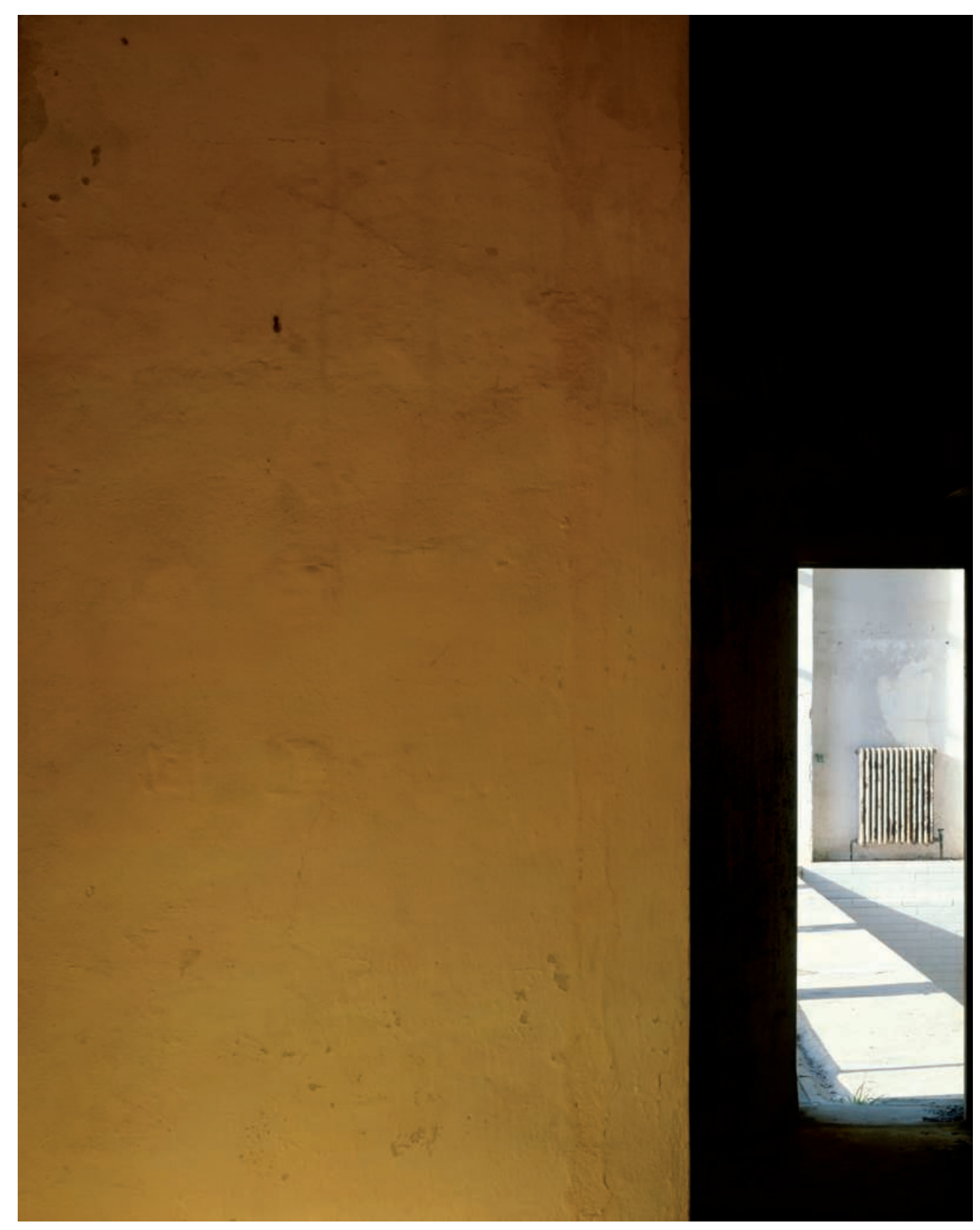

Fig. 12 : Villa Noailles, Hyères, 1996 (Ilfochrome, $130 \times 104 \mathrm{~cm}, 3$ exemplaires) (C) Stéphane Couturier 